\title{
Retrospective analysis of laboratory testing in 54 patients with severe- or critical-type 2019 novel coronavirus pneumonia
}

\author{
Yanlei $\mathrm{Li} \mathbb{1}^{1} \cdot$ Yanbing $\mathrm{Hu}^{2} \cdot$ Jinyan $\mathrm{Yu}^{3} \cdot$ Tiangang $\mathrm{Ma}^{3}$ \\ Received: 11 March 2020 / Revised: 7 April 2020 / Accepted: 7 April 2020 / Published online: 27 April 2020 \\ (c) The Author(s), under exclusive licence to United States and Canadian Academy of Pathology 2020
}

\begin{abstract}
Timely analysis of the laboratory characteristics associated with 2019 novel coronavirus pneumonia (COVID-19) can assist with clinical diagnosis and prognosis. This study is a collection of clinical data from 54 hospitalized adult patients diagnosed with COVID-19 in the Zhongfa Xincheng district of China at Tongji Hospital of Huazhong University of Science and Technology from January 28, 2020 to February 11, 2020. The average age of the patients was $61.8 \pm 14.5$ years, and the predominant age group was 50-79. The proportion of critical-type patients with comorbidities was higher than that of severe-type patients. Lymphocyte counts were significantly reduced in routine bloodwork for all patients, but significantly lower in critical-type patients than that in severe-type patients. Prolongation of prothrombin times (PT) and elevation of fibrinogen degradation products (FDPs) and D-dimers (D-Ds) were detected in coagulation function tests, and more significant changes were observed in critical-type patients compared to severe-type patients. Serum ferritin levels were sensitive to severe acute respiratory syndrome coronavirus 2 (SARS-CoV-2) infection but could not be used for disease assessment. In addition, levels of two inflammatory factors, soluble interleukin-2 receptor (sIL-2R) and interleukin-6 (IL-6) were significantly increased in all patients, but higher in critical-type patients than in severe-type patients. Moreover, kidney injury was the second-most common organ affected by COVID-19 followed by heart and liver. Kidney and heart injury were more severe in critical-type patients than in severe-type patients. All of the 31 severe-type patients recovered. Of the criticaltype patients, six died and 17 recovered. The length of hospital stay for critical-type patients was significantly longer for severe-type patients. In summary, increased lymphocyte counts, prolonged PT, secondary increases in fibrinolytic activity and increases in sIL-2R and IL-6 are typical features of COVID-19 and are associated with disease severity.
\end{abstract}

\section{Introduction}

In December 2019, COVID-19, which is pneumonia caused by the 2019 novel coronavirus, was first found in Wuhan, Hubei province, China. It spread rapidly to other provinces in China and internationally. It is a new infectious disease, which primarily causes respiratory tract infection, but also

Tiangang Ma

Matg2001@163.com

1 Department of Clinical Laboratory, The Second Hospital of Jilin University, 130041 Changchun, Jilin, People's Republic of China

2 Department of Ultrasound, The Second Hospital of Jilin University, 130041 Changchun, Jilin, People's Republic of China

3 Department of Respiratory and Critical Care Medicine, The Second Hospital of Jilin University, 130041 Changchun, Jilin, People's Republic of China can lead to multiple organ dysfunction, most notably damage to the kidney, liver, and other organs. In this study, we summarize the results of routine laboratory testing of both severe-type and critical-type COVID-19 in order to define practical indicators for the diagnosis and treatment of COVID-19.

\section{Methods}

\section{Patients}

This study is a collection of clinical data from 54 adult patients diagnosed with COVID-19 that were hospitalized at Wuhan Tongji Hospital, Huazhong University of Science and Technology, from January 28 to February 11, 2020. The standard reference for diagnosis, clinical classification, and treatment of COVID-19 was developed by the National Health Commission of the People's Republic of China 

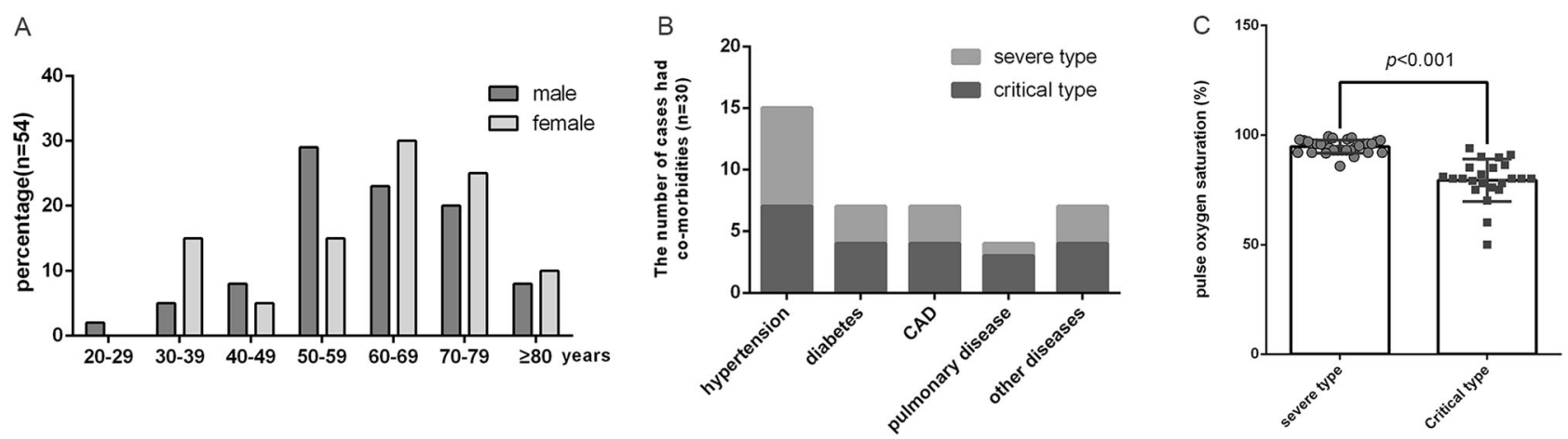

Fig. 1 General characteristics of patients. The ages of the patients (a), the distribution of comorbidities in patients (b). pulse oxygen saturation at the time of admission (c). CAD coronary artery heart disease; $n=54$.

(version 4) [1]. All patients were positive for severe acute respiratory syndrome coronavirus 2 (SARS-cov-2) nucleic acid as measured by real-time fluorescence reverse transcription polymerase chain reaction (RT-PCR) in respiratory tract samples. The patients exhibited fever and respiratory symptoms and pneumonia was diagnosed by imaging. The diagnosis was determined as severe-type if one of the following conditions were present: respiratory distress; respiratory frequency $\geq 33$ times/min; oxygen saturation less than $93 \%$ in a resting state; or partial arterial oxygen pressure $\left(\mathrm{PaO}_{2}\right)$ /oxygen absorption concentration $\left(\mathrm{FiO}_{2}\right) \leq 300 \mathrm{mmHg}(1 \mathrm{mmHg}=0.133 \mathrm{kpa})$. Any of the following conditions resulted in a diagnosis of critical-type: respiratory failure requiring mechanical ventilation; shock; or transfer to the intensive care unit (ICU) due to other organ failure. The respiratory support treatment for severetype patients was medium-flow nasal cannula $(5 \mathrm{~L} / \mathrm{min})$, critical-type patients received noninvasive ventilation (NIV), and/or invasive mechanical ventilation (IMV). Recovery was also defined by National Health Commission of the People's Republic of China and include the disappearance of clinical symptoms, two consecutive negative nucleic acid tests for SARS-CoV-2 (sampling time interval at least $24 \mathrm{~h}$ ), and absence of viral pneumonia as determined by a computed tomography (CT) scan of the chest.

\section{Data collection}

Patient data were collected through the hospital's electronic medical record system and included general personal information, previous medical history, and laboratory testing. Blood coagulation function, blood biochemistry, and measurement of inflammatory factors were performed within $24 \mathrm{~h}$ of admission. Ferritin and C-reactive protein (hs-CRP) levels were determined within 15 days of admission. Pulse oxygen saturation data were collected immediately upon admission.

\section{Statistical analysis}

SPSS Statistics software ver.19.0 (IBM Co. Armonk, NY, USA) was used for analyzing data. The data with a normal distribution were expressed as mean \pm standard deviation (SD) and assessed by Student's $t$-test. The counted data were expressed as a percentage and compared by Chisquare test. $P<0.05$ was considered statistically significant.

\section{Results}

\section{Basic characteristics of patients}

The average age of the patients enrolled in this study was $61.8 \pm 14.5$ years, with the age group ranging from 50 to 79 years old as the predominant population (Fig. 1a). A total of 30 patients $(55.6 \%)$ had comorbidities, including hypertension (15), diabetes (7), coronary artery heart disease (CAD) (7), pulmonary disease (4), and other diseases (7); among those, 10 patients exhibited two distinct pre-existing conditions (Fig. 1b); Among the 54 patients, 34 were males (63\%), of which 19 had comorbidities (55.9\%). There were 20 females (37\%), 11 with comorbidities $(55 \%)$, and there was no significant difference in the proportion of males and females with comorbidities (Table 1). Among the 54 patients, 31 (57\%) were severe-type, while $23(43 \%)$ were critical-type. There were more patients in the critical-type group with comorbidities than severe-type group (Table 2). Pulse oxygen saturation at the time of admission was higher in severe-type patients than in critical-type patients, as shown in Fig. 1c.

\section{Blood and coagulation analysis}

Of the 54 patients, neutrophil counts within the reference range $\left(1.8-6.3 \times 10^{9} / \mathrm{L}\right)$ were found in 26 cases $(48.1 \%) ; 21$ cases were above the upper limit (38.9\%), and seven cases 
Table 1 Gender differences in comorbidities.

\begin{tabular}{lcccc}
\hline & Male & Female & Total & $P$ \\
\hline Comorbidities & 19 & 11 & 30 & \\
No comorbidities & 15 & 9 & 24 & \\
Total & 34 & 20 & 54 & 0.950 \\
\hline
\end{tabular}

Table 2 Differences between severe-type and critical-type patients with comorbidities.

\begin{tabular}{llcll}
\hline & Severe-type & Critical-type & Total & $P$ \\
\hline Comorbidities & 12 & 18 & 30 & \\
No comorbidities & 19 & 5 & 24 & \\
Total & 31 & 23 & 54 & 0.004 \\
\hline
\end{tabular}

were below the lower limit (13\%) (Fig. 2a). The neutrophil counts in males were higher than those of females (Fig. 2b), and were also higher in the critical-type group than that in severe-type group (Fig. 2c). Lymphocyte counts within the reference range $\left(1.1-3.2 \times 10^{9} / \mathrm{L}\right)$ were seen in seven cases $(13 \%)$, none were above the upper limit of the reference range, and 47 cases were below the lower limit of the reference range (87\%) (Fig. 2d). There were no differences in lymphocyte counts between males and females (Fig. 2e), but the critical-type patients had lower lymphocyte counts than the severe-type patients (Fig. 2f). Of 53 patients, there were $27(50.9 \%)$ patients with prolonged prothrombin (PT) and $12(22.6 \%)$ patients with prolonged activated partial thromboplastin time (APTT). One patient was excluded from PT analysis after aortic dissection, because cardiac surgery can affect clotting function. In critical-type patients, PT was longer than that in severe-type patients (Fig. 2g), while APTT showed no statistical difference between the two groups (Fig. 2h). The levels of fibrinogen degradation products (FDPs) above the reference range $(<5.0 \mu \mathrm{g} / \mathrm{mL})$ for critical-type patients was higher than that of severe-type patients (Table 3). There were 46 patients (86.8\%) with levels of D-dimers (D-Ds) above the reference range $(<0.5 \mathrm{~g} / \mathrm{mL} \mathrm{FEU})$; among those, the proportion of criticaltype patients was higher than that for severe-type patients (Table 4).

\section{Ferritin levels}

In the 54 patients, ferritin levels above the upper limit of the reference range ( $15-150 \mathrm{~g} / \mathrm{L}$ for females and $30-400 \mathrm{~g} / \mathrm{L}$ for males) were detected in $49(90.7 \%)$ patients (Fig. 3a). We selected four patients who were continuously monitored for ferritin and hs-CRP for 10-15 days. Ferritin levels decreased as hs-CRP decreased, but were significantly higher than the upper reference range for at least 5 days after hs-CRP returned to normal (Fig. 3b).

\section{Inflammatory cytokines}

Forty-seven patients were examined for inflammatory cytokines within $24 \mathrm{~h}$ of admission. Inflammatory cytokines were detected including iterleukin-1 (IL-1), soluble IL-2 receptor (sIL-2R), IL-6, IL-8, IL-10, and tumor necrosis factor- $\alpha$ (TNF- $\alpha)$. The number of patients with cytokine levels above the upper limit of the reference range were 6 , $39,35,6,20$, and 37, respectively (Fig. 4a). The concentrations of sIL-2R and IL- 6 in critical-type patients were significantly higher than those in severe-type patients (Fig. $4 \mathrm{~b}$ and c), while the levels of IL-10 and TNF- $\alpha$ were not statistically different between the two groups (Fig. $4 \mathrm{~d}$ and e).

\section{Organ damage}

The estimated glomerular filtration rate (eGFR) is an indicator of renal injury calculated by the Chronic Kidney Disease Epidemiology Collaboration (CKD-EPI) equation. eGFR values below the lower limit of the reference range indicate renal injury. High sensitivity Troponin I (hsTnI) was used as a heart injury marker and alanine aminotransferase (ALT) was used as a liver injury marker; values above the upper limit of the reference ranges are signs of heart injury and liver injury.

Kidney injury (Fig. 5a) and heart injury (Fig. 5b) in the critical-type patients were more serious than in severe-type patients, but there was no statistical difference in liver injury between the two groups (Fig. 5c). Among the 54 patients, 41 patients were observed $(75.9 \%)$ with organ damage, including 29 patients $(70.7 \%)$ with kidney injury, 23 patients $(56.1 \%)$ with heart injury, and 17 patients (31.5\%) with liver injury (Fig. 5d).

\section{Disease outcomes}

All of the 31 severe-type patients recovered. Six criticaltype patients died and 17 recovered. The length of the hospital stays were $21.4 \pm 6.5$ days for severe-type patients and $29.3 \pm 6.9$ days for critical-type patients who recovered. The lengths of hospital stays for critical-type patients was significantly longer than that of severe-type patients (Fig. 6).

\section{Discussion}

All patients in this study were adults. Among the 54 severe/ critical-type patients, the average age was $61.8 \pm 14.5$ years, 

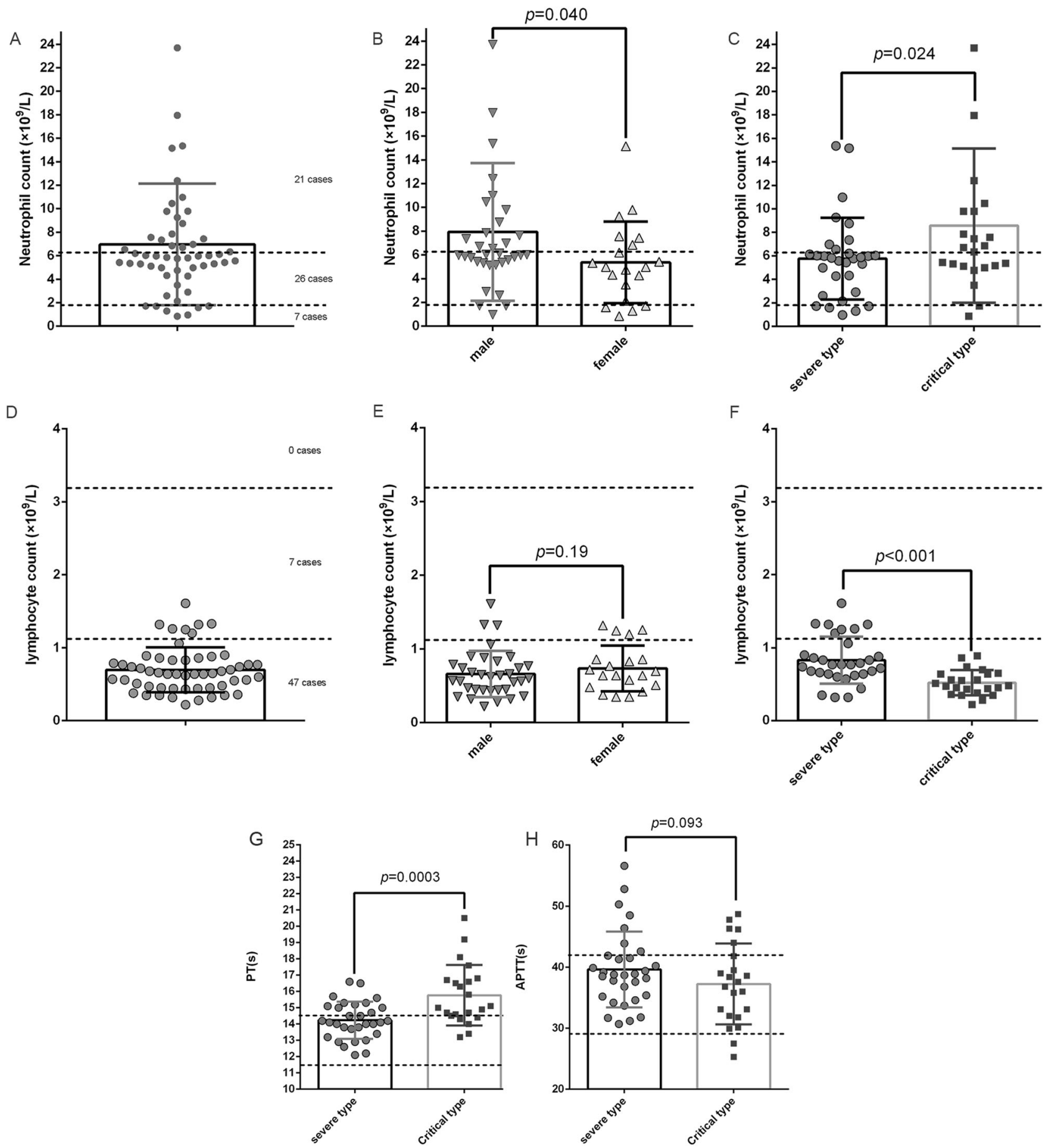

Fig. 2 Bloodwork and coagulation function testing. Neutrophil counts in all patients (a); comparison of neutrophil counts in males and females (b); comparison of neutrophil counts in severe-type and critical-type patients (c); lymphocyte counts in all patients (d); comparison of lymphocyte counts in males and females (e); comparison of

with the predominant population in the age group of 50-79 years. The relationship between the age and severity of disease may be due to weaker immune systems and more comorbidities in older patients. Therefore, people with comorbidities, such as hypertension, diabetes, coronary lymphocyte count in severe-type and critical-type patients (f); comparison of PT values of severe-type and critical-type patients (g); comparison of APTT values of severe-type and critical-type patients (h). PT prothrombin, APTT activated partial thromboplastin time; The two dotted lines are the upper and lower limits of the reference range.

heart disease, and the elderly, appear to have a higher risk for COVID-19.

All 54 patients showed significant reductions in lymphocyte counts, but the critical-type patients had lower counts than the severe-type. There was no significant 
Table 3 Comparison of FDPs test between severe-type and critical-type patients.

\begin{tabular}{lllll}
\hline & Severe-type & Critical-type & Total & $P$ \\
\hline Within the reference range & 21 & 3 & 24 & \\
Above the reference range & 10 & 19 & 29 & \\
Total & 31 & 22 & 53 & $<0.001$ \\
\hline
\end{tabular}

Table 4 Comparison of D-D test between severe-type and critical-type patients.

\begin{tabular}{lcccc}
\hline & $\begin{array}{l}\text { Severe- } \\
\text { type }\end{array}$ & $\begin{array}{l}\text { Critical- } \\
\text { type }\end{array}$ & Total & $P$ \\
\hline Within the reference range & 7 & 0 & 7 & \\
Above the reference range & 24 & 22 & 46 & \\
Total & 31 & 22 & 53 & 0.003 \\
\hline
\end{tabular}

difference between males and females. The decreases in lymphocytes in peripheral blood may have been caused by SARS-CoV-2 infection, which may have led to apoptosis of lymphocytes and phagocytosis by immune cells. Alternatively, lymphocytes may have been stimulated by inflammatory cytokines, penetrated the blood vessels and accumulated in tissue. A recent study [2] found that CD4+ and CD8+ T lymphocytes in peripheral blood of patients with COVID-19 were significantly reduced, but the Th17 subset in CD4+ T lymphocytes were increased, and $\mathrm{CD} 8+\mathrm{T}$ cells contained higher concentrations of cytotoxic particles. These results indicate that the SARS-CoV-2 might seriously impair the immune system. Further experimental data are required, such as peripheral blood flow cytometry, analysis of humoral immunity, and examination of bone marrow to determine the exact effect of the virus on the immune system. In addition, we found that high neutrophil counts were seen in $38.9 \%$ of the 54 patients and were higher in critical-type patients than that in severe-type patients. One explanation for this observation may be that more critical-type patients had bacterial co-infections. Prolongation of PT and elevation of D-Ds and FDPs were the main characteristics of the abnormal coagulation, but APTT was not significantly changed. We hypothesized that SARS$\mathrm{CoV}-2$ infection can cause tissue damage, resulting in the release of tissue factors, which promote the exogenous coagulation pathway, leading to prolonged PT. Increases in D-Ds and FDPs indicate that a patient is in a state of secondary fibrinolysis hyperactivity. According to the COVID19 treatment regimen developed by the National Health Commission, People's Republic of China (trial version 7) ${ }^{1}$, SARS-CoV-2 infection can lead to partial vascular endothelial shedding and thrombosis. We believe that thrombosis might cause secondary increased fibrinolysis activity and increased D-Ds and FDPs.

Serum ferritin elevation may occur during infection and inflammation. This study shows that ferritin levels were significantly elevated in severe/critical-type COVID-19 patients and was the last laboratory value to return to normal. In contrast, Hs-CRP, an acute temporal protein with a half-life of $\sim 19 \mathrm{~h}$, returned to normal levels at least 5 days before ferritin did. A previous study [3] has shown that the most common cause of serum ferritin elevation is non-human immunodeficiency virus infection. We believe that the SARS-CoV-2 infection caused the increases in serum ferritin, which did not change as patients improved, and therefore cannot be used for disease assessment. However, another study [4] has determined that assembly of Middle East Respiratory Syndrome (MERS) coronavirus nanoparticles is related to a chaperone-mediated ferritin. The specific relationship between ferritin and coronavirus infection in humans therefore requires further study.

After a virus infects human body, immune cells release cytokines. Continuous infection by the virus may cause uncontrolled and excessive amplification of inflammatory factors, forming a "cytokine storm", causing multiple organ damage and dysfunction. IL-6 may be a reliable biomarker for the severity and prognosis of a cytokine storm, and a better indicator that TNF- $\alpha$ or IL-1 [5, 6]. In this study, sIL$2 \mathrm{R}$, IL- 6 and TNF- $\alpha$ were significantly elevated in the sera of severe- and critical-type COVID-19 patients. In addition to IL-6, sIL-2R was also significantly increased and positively correlated with disease severity. Therefore, sIL-2R may be a prognostic indicator or a target for treating inflammation. TNF- $\alpha$ and IL-10 were also elevated, but there was no significant difference between levels of these cytokines between the severe-type and critical-type patients. It has been reported that IL-1 plays an important role in the cytokine storms seen in severe influenza, but we found that the proportions of IL-1 and IL-8 elevation in both types of COVID-19 were very low.

SARS-CoV enters lung cells through angiotensinconverting enzyme 2 (ACE2) after infection [7]. In addition to lung tissue, ACE2 is also highly expressed in vascular endothelial cells, heart, kidney and liver. Studies have reported that SARS-CoV can cause myocardial injury and lead to myocardial inflammation [8]. Pathologic findings in patients with COVID-19 showed inflammatory cell infiltration in the myocardium [2]. There have also been reports of detection of SARS-CoV in distal convoluted renal tubules and livers of patients with SARS [9]. Most studies of organ damage by coronavirus focus SARS-CoV, little is 

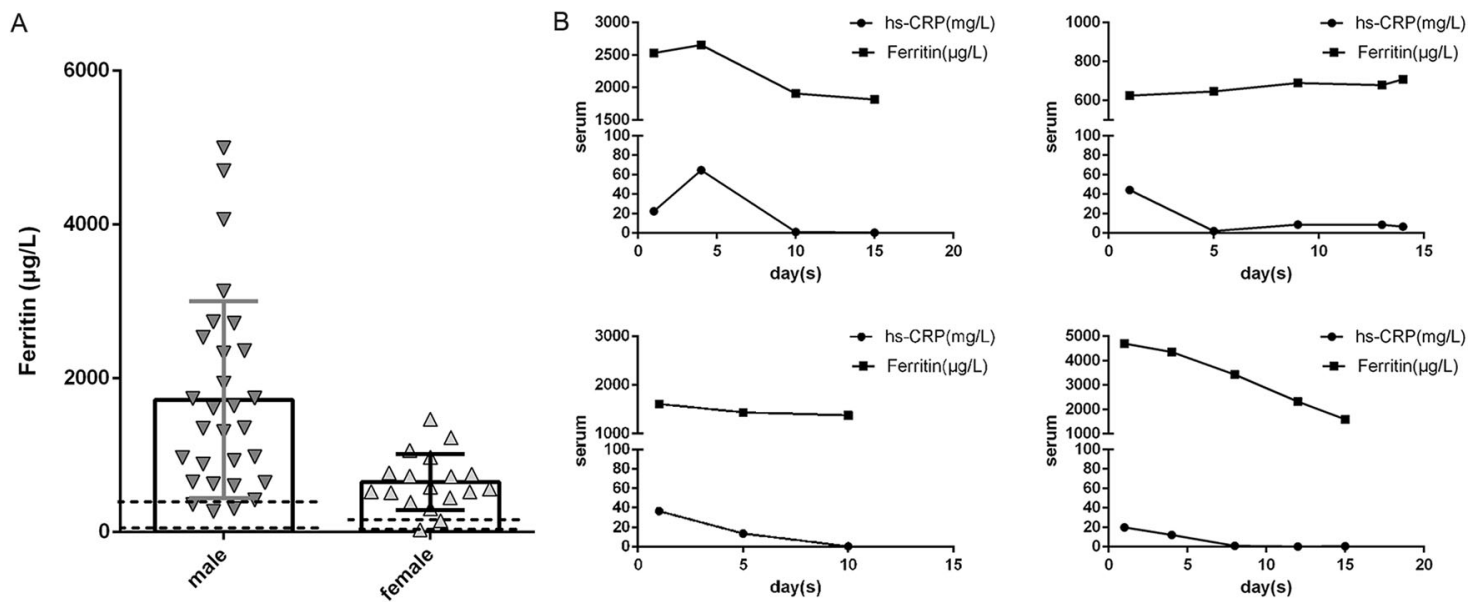

Fig. 3 Detection of serum ferritin. Serum ferritin measurement in all patients (a); duration of the increase in ferritin and hs-CRP (b). hs-CRP hypersensitive c-reactive protein. The two dotted are the upper and lower limits of the reference range.

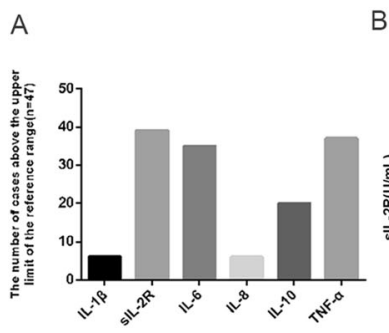

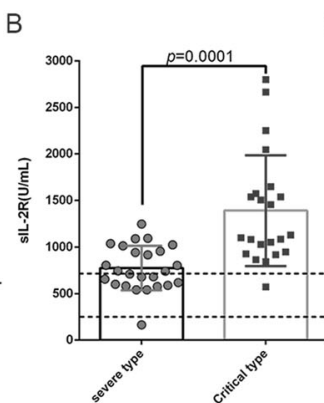

C

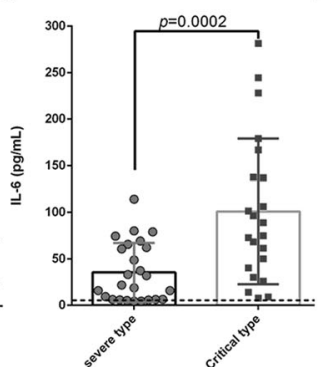

D

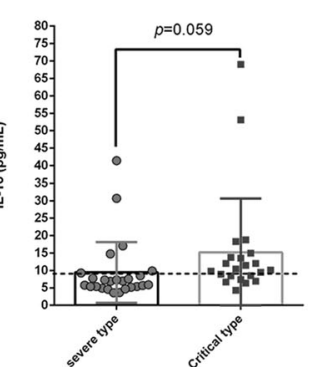

E

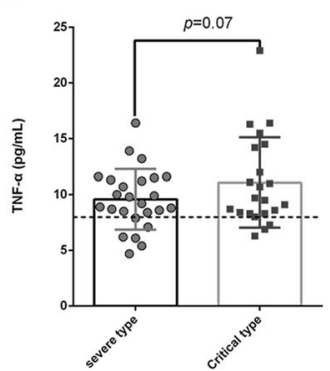

Fig. 4 The detection of inflammatory factors. Frequency of inflammatory factor levels above the upper limit of the reference range (a); sIL-2R level in severe and critical-type patients (b); IL-6 level in severe and critical-type patients (c); IL-10 levels in severe and critical- type patients (d); TNF- $\alpha$ concentrations in severe and critical-type patients (e). sIL-2R: soluble IL-2 receptor; $(n=47)$. The two dotted lines are the upper and lower limits of the reference range for detection, The single dotted line is the upper limit of the reference range.
A

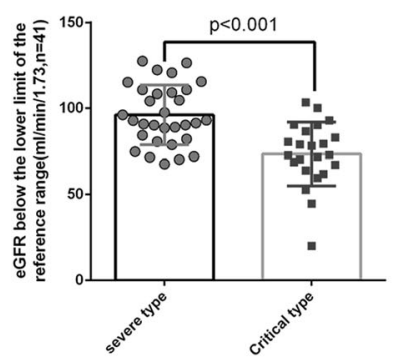

B

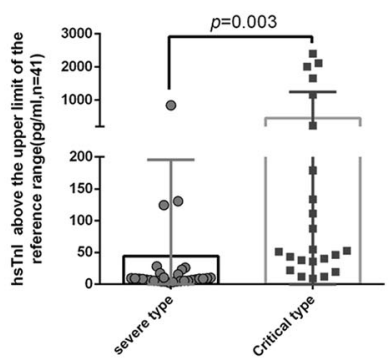

Fig. 5 Blood biochemistry. Comparison of the estimated glomerular filtration rate (eGFR) in severe-type and critical-type patients (a), Comparison of high sensitivity Troponin hsTnI (b), and alanine

known about the effects of SARS-CoV-2. In this study, we summarized the frequency of organ injury outside the lung and found that the kidney injury (as assessed by eGFR) was most common, followed by the heart injury (as assessed by hsTnI) and the liver injury (as assessed by ALT). Kidney and heart injury were more severe in critical-type patients
C
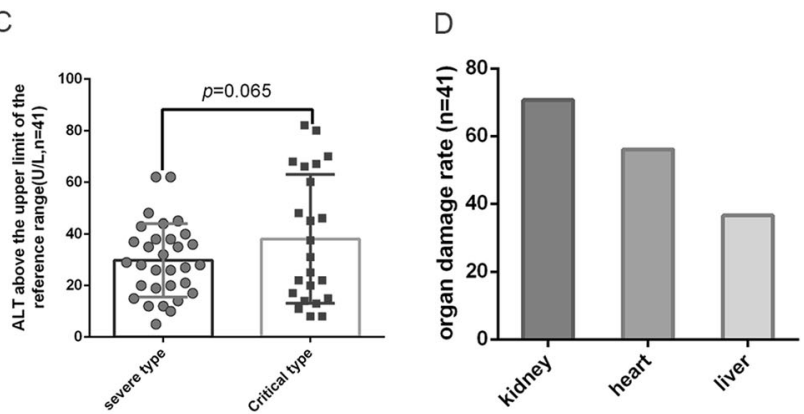

aminotransferase ALT (c) between severe and critical-type patients, Histogram of the number of cases of organ injury $(\mathbf{d})$. $(n=41)$.

than in severe-type patients, suggesting that critical-type patients are more likely to have multiple organ injuries.

Compared with critical-type patients, severe-type patients have a better prognosis and a high rate of recovery because they exhibit high pulse oxygen saturation, do not need noninvasive ventilation (NIV) and/or invasive mechanical 


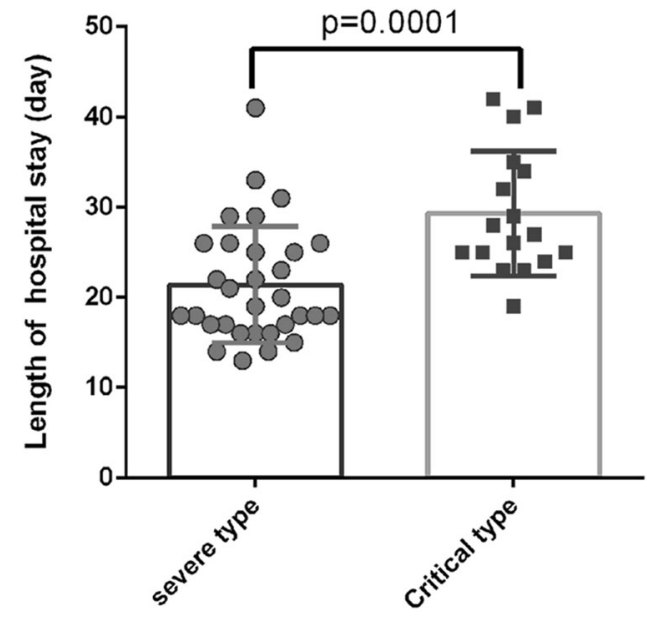

Fig. 6 The lengths of hospital stays. The lengths of hospital stays for severe and critical-type patients $(n=48)$.

ventilation (IMV), experience less intense inflammatory cytokine storms, and show less organ damage.

In conclusion, this study is a single-center, retrospective case study and is limited to general data and laboratory tests of critical-type and severe-type patients. We hope that the conclusions of this study can provide assistance for clinicians in the diagnosis, treatment, and prognosis of COVID-19.

\section{Compliance with ethical standards}

Conflict of interest The authors declared that they have no conflict of interest.

Ethical approval The ethics committee of the second hospital of Jilin university approved the collection and usage of the relevant data.
Publisher's note Springer Nature remains neutral with regard to jurisdictional claims in published maps and institutional affiliations.

\section{References}

1. Magnus MC, Haberg SE, Stigum H, Nafstad P, London SJ, Vangen $S$, et al. Delivery by Cesarean section and early childhood respiratory symptoms and disorders: the Norwegian mother and child cohort study. Am J Epidemiol. 2011;174:1275-85.

2. Xu Z, Shi L, Wang Y, Zhang J, Huang L, Zhang C, et al. Pathological findings of COVID-19 associated with acute respiratory distress syndrome. Lancet Respir Med. 2020;8:420-2.

3. Senjo H, Higuchi T, Okada S, Takahashi O. Hyperferritinemia: causes and significance in a general hospital. Hematology. 2018;23: 817-22.

4. Kim YS, Son A, Kim J, Kwon SB, Kim MH, Kim P, et al. Chaperna-mediated assembly of ferritin-based middle east respiratory syndrome-coronavirus nanoparticles. Front Immunol. 2018; 9:1093.

5. Tanaka T, Narazaki M, Kishimoto T. Immunotherapeutic implications of IL-6 blockade for cytokine storm. Immunotherapy. 2016; 8:959-70.

6. Tanaka T, Narazaki M, Kishimoto T. IL-6 in inflammation, immunity, and disease. Cold Spring Harb Perspect Biol. 2014;6: a016295.

7. Song W, Gui M, Wang X, Xiang Y. Cryo-EM structure of the SARS coronavirus spike glycoprotein in complex with its host cell receptor ACE2. PLoS Pathog. 2018;14:e1007236.

8. Oudit GY, Kassiri Z, Jiang C, Liu PP, Poutanen SM, Penninger JM, et al. SARS-coronavirus modulation of myocardial ACE2 expression and inflammation in patients with SARS. Eur J Clin Invest. 2009;39:618-25.

9. Ding Y, He L, Zhang Q, Huang Z, Che X, Hou J, et al. Organ distribution of severe acute respiratory syndrome(SARS) associated coronavirus(SARS-CoV) in SARS patients: implications for pathogenesis and virus transmission pathways. J Pathol. 2004;203: 622-30. 